\title{
LETTER \\ A Compact Loop Antenna System for Monitoring Local Electromagnetic Environments in Geospace
}

\author{
Satoshi YAGITANI $^{\dagger a}$, Mitsunori OZAKI ${ }^{\dagger}$, and Hirotsugu KOJIMA $^{\dagger \dagger}$, Members
}

\begin{abstract}
SUMMARY A sensor network consisting of a number of palm-sized nodes with small electric and magnetic sensors has been proposed to monitor local electromagnetic activities in space plasmas. In the present study, a compact loop antenna system is designed and fabricated for use in sensor nodes that can capture magnetic vector fields from ELF to MF frequencies. The performance of the developed system is shown to be sufficient to allow measurement of the magnetic field activity around artificial structures in addition to intense natural plasma waves in geospace.

key words: loop antenna, sensor network, electromagnetic environment, space plasma
\end{abstract}

\section{Introduction}

In-situ plasma wave observations by scientific satellites are essential for understanding the dynamics and structure of space plasma regions such as the magnetosphere and the ionosphere around the Earth (so-called "geospace"), where kinetic energy is generated and transferred via plasma waves in collisionless plasmas. Plasma waves have a wide frequency range, from ULF (lower than several $\mathrm{Hz}$ ) up to $\mathrm{HF}$ (higher than $\mathrm{MHz}$ ), depending on the ion and electron dynamics, and can be either electrostatic or electromagnetic in nature. They can remain extremely faint for extended periods of time, representing only the thermal motion of plasma particles, and then become enhanced by several orders of magnitude due to instabilities. Conventional plasma wave receivers carried on board satellites have comprised sensitive but bulky electric and magnetic sensors and low-noise preamplifiers, with wideband and wide-dynamicrange characteristics. Such receivers usually involve largescale and highly complex electronic circuits [1]. Recently, a miniaturized plasma wave receiver has been proposed and developed for use in future space science missions involving miniaturized satellites, such as formation-flight satellites [2]. In the proposed device, the main functional blocks of the analog electronics for the wideband waveform receiver, such as filters and amplifiers, which had conventionally been fabricated on a large printed circuit board (PCB) using discrete components, were integrated using an analog-specificintegrated-circuit (ASIC) chip. Six receivers (3 for electric and 3 for magnetic field vectors) were integrated into a

Manuscript received October 15, 2010.

Manuscript revised January 4, 2011.

$\dagger$ The authors are with Kanazawa University, Kanazawas-shi, 920-1192 Japan.

${ }^{\dagger}$ The author is with the Research Institute of Sustainable Humanosphere, Kyoto University, Uji-shi, 611-0011 Japan.

a) E-mail: yagitani@ @eg.is.t.kanazawa-u.ac.jp

DOI: 10.1587/transcom.E94.B.1744
$3 \mathrm{~mm}$ square ASIC chip. Such a configuration drastically reduces the overall size and mass of the onboard plasma wave receiver. As an application of such miniaturized plasma wave receivers, a space sensor network system, referred to as a "monitor system for space electromagnetic environments (MSEE)," has previously been proposed [2]. The system consists of a number of palm-sized sensor nodes distributed throughout the target area of space, each of which carries a compact plasma wave receiver with small electric and magnetic sensors. This sensor network is designed to monitor electromagnetic activity simultaneously at multiple points in the vicinity (within $\mathrm{km}$ ) of space plasmas. For instance, plasma wave turbulence was found to be generated in a region of several hundred meters surrounding the space shuttle, where both electrostatic and electromagnetic waves were observed [3], [4]. Knowledge concerning the structure of such turbulence is crucial for understanding the plasma dynamics around artificial structures, particularly in a space environment. The palm-sized MSEE sensor nodes must be equipped with small but sensitive electric and magnetic sensors, that are capable of monitoring the weakest fields possible. In the present study, we have developed a compact loop antenna system to be installed in an MSEE node, to detect magnetic vector fields. Its target frequency range is from ELF (hundreds of $\mathrm{Hz}$ ) to MF (hundreds of $\mathrm{kHz}$ ), corresponding to the plasma wave turbulence observed in the vicinity of the space shuttle in the ionosphere [3], [4]. The performance of the loop antenna system is confirmed to be sufficiently high to render it capable of measuring magnetic fields associated with plasma wave turbulence around space structures, in addition to intense natural plasma waves in geospace.

\section{MSEE Sensor Node}

A fabricated prototype of an MSEE sensor node is shown in Fig. 1 [2]. The main body is a $7-\mathrm{cm}$ cubic chassis containing preamplifiers, wideband receivers packed in an ASIC chip, a digital processing unit, a wireless communication module and batteries. The chassis is made of aluminum covered with magnetic-material panels. These provide electrostatic and magnetic shielding to reduce leakage of internal noise emissions from the electronics, which would otherwise be picked up by the electric and magnetic sensors installed outside of the main body. Electric fields are measured by three orthogonal dipole antennas with tip-to-tip distances of $40 \mathrm{~cm}$, which are fabricated using six top-hat monopole an- 


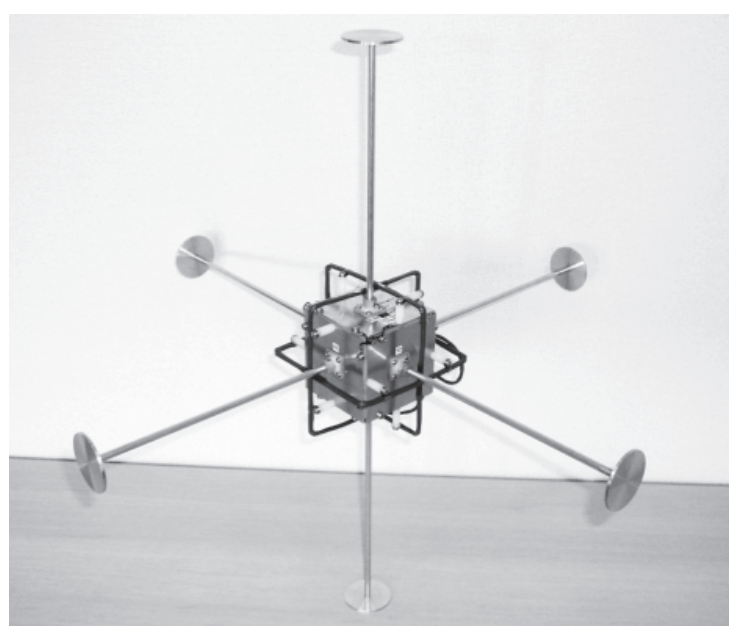

Fig. 1 Prototype of MSEE sensor node.

tennas protruding from the six surfaces of the cubic body. On the other hand, magnetic fields are picked up by three orthogonal loop antennas, each of which is $10-\mathrm{cm}$ square and encloses the main body, as explained in detail in Sect. 3. Thus, all six electromagnetic vector components are measured by these sensors.

\section{Compact Magnetic Sensor for MSEE Node}

The typical magnetic sensors carried on board satellites are induction-type devices such as search coils and loop antennas [1], which have been used for decades because of their high sensitivity, stability and robustness even in space plasma environments. In practice, search coils are used for lower frequencies (less than 10 to $20 \mathrm{kHz}$ ), whereas loop antennas are used for higher frequencies (up to $\mathrm{MHz}$ ). Here we adopt a loop antenna as the magnetic sensor in the MSEE node, to measure magnetic fluctuations between ELF and MF frequencies in the ionosphere. At ionospheric altitudes of a few hundred kilometers, the electron cyclotron frequency is of the order of $1 \mathrm{MHz}$, below which whistler-mode waves with large magnetic field components are excited and propagate. In fact, strong electromagnetic whistler-mode emissions with frequencies of up to about $1 \mathrm{MHz}$ were generated by an electron beam emitted from the space shuttle, and were observed within several hundred meters of the shuttle [4].

Three loop antennas are arranged orthogonally, and enclose the MSEE body as shown in Fig. 2(a), to measure the three axial components of the magnetic field vector. Each loop, which consists of 15 turns of insulated copper wiring, is $10-\mathrm{cm}$ square. Figure 2(b) shows a block diagram of the loop antenna and its preamplifier for each of the three axes. The loop is wrapped in a copper foil grounded to the MSEE chassis, which acts as an electrostatic shield to prevent electric field pick-up. A slit is made in the wrapping so that a shorted turn is not created. The voltage induced at the loop terminal is then fed to a preamplifier located inside the main body. The preamplifier for each axial loop was designed

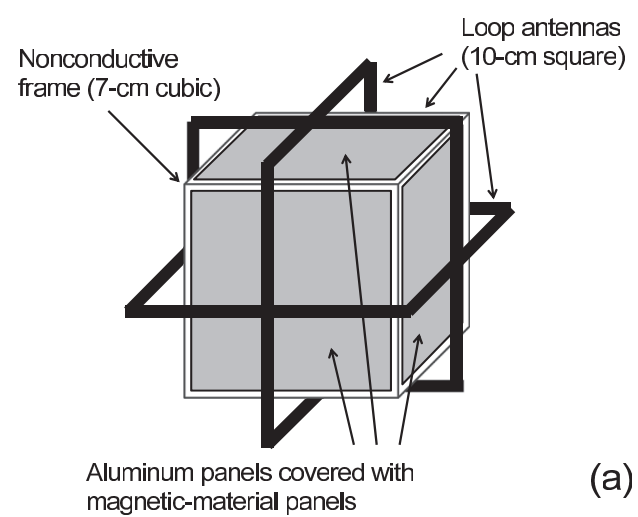

(a)

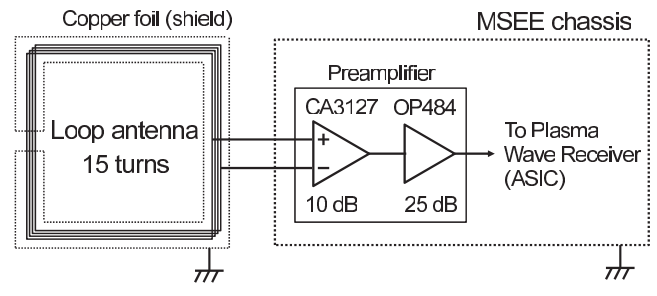

(b)

Fig. 2 Configuration of loop antenna system in MSEE node. (a) Tri-axial loop antennas arranged around the MSEE body, and (b) Block diagram of each axial antenna and its preamplifier.

to have low input voltage noise (about $1 \mathrm{nV} / \mathrm{Hz}^{1 / 2}$ ) to eliminate the need for the step-up transformers normally used with loop antennas [1]. Small discrete chip elements were used to make the preamplifier circuit as compact as possible. By constructing the 1st-stage differential amplifier using low-noise transistors (CA3127), and the 2nd-stage gainadjustment amplifier using a low-noise operational amplifier (OP484), both of which were powered by a single $3.3 \mathrm{~V}$ supply, the total PCB area occupied by the three preamplifiers is smaller than 5-cm square and so can fit within the body of the MSEE node. The combined power consumption of the three preamplifiers is $32 \mathrm{~mW}$.

Following fabrication, the loop antennas were installed in a prototype MSEE node, as shown in Fig. 1. The electrical performance of the antennas was then measured in a magnetically shielded room, in which a reference magnetic field ( $1 \mathrm{nT})$ was created by a Helmholtz coil 2 meters in diameter. Figure 3 shows the performance of an isolated loop antenna (i.e., an air-core loop without the MSEE body inserted), for one of the three orthogonal axes; the antennas for the other two axes exhibited almost the same performance. Figure 3(a) shows the magnetic gain as a function of frequency, which is defined as the output voltage of the preamplifier when the magnetic field of $1 \mathrm{nT}$ is applied to the loop sensor. The measured gain (solid line) agrees well with the theoretical gain (dotted line) calculated using a circuit simulator (Micro-Cap 8.0, Spectrum Software). The gain linearly increases with frequency from $300 \mathrm{~Hz}$ up to around $100 \mathrm{kHz}$, as expected from Faraday's law of induction. A peak is observed around $700 \mathrm{kHz}$ due to parallel resonance 

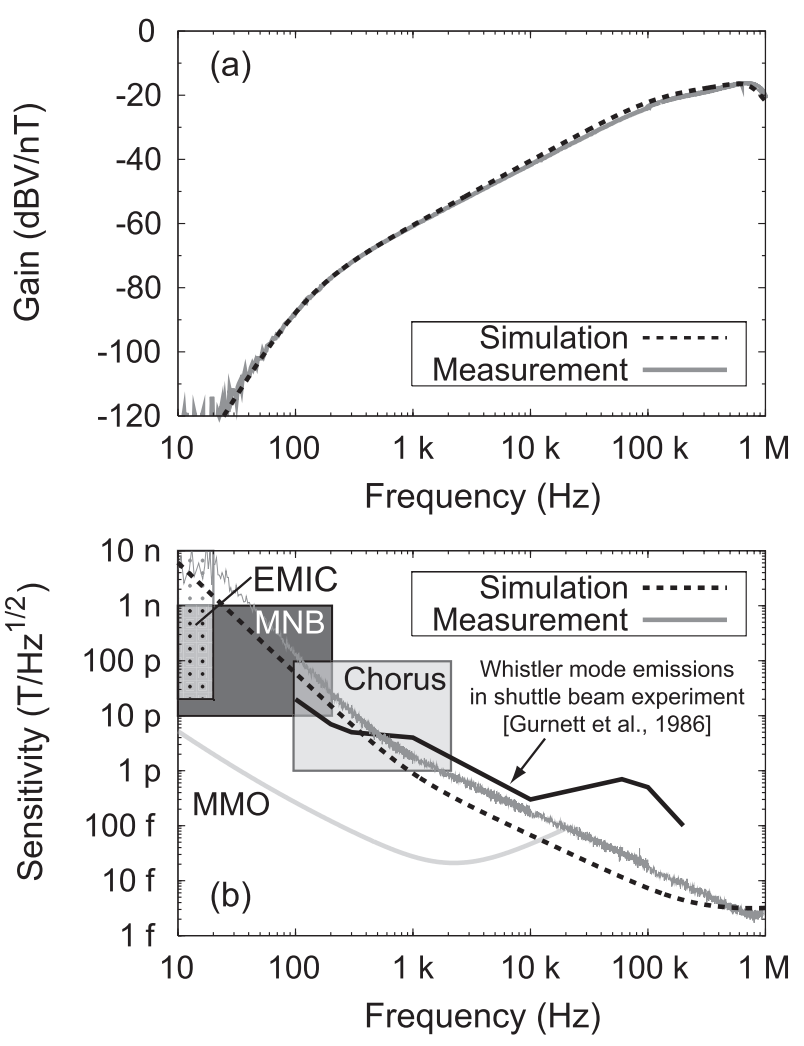

Fig. 3 Loop antenna performance. (a) gain, and (b) sensitivity.

between the inherent inductance and the stray capacitance of the loop antenna. In Fig. 3(b), the measured and simulated magnetic sensitivities are plotted as solid and dotted lines, respectively. The measured sensitivity is seen to be lower than the simulated value by up to about $10 \mathrm{~dB}$, which is probably due to the real transistors and operational amplifiers having higher noise levels than those implemented in the simulation. The measured sensitivities were $2 \mathrm{pT} / \mathrm{Hz}^{1 / 2}$, $200 \mathrm{fT} / \mathrm{Hz}^{1 / 2}$ and $20 \mathrm{fT} / \mathrm{Hz}^{1 / 2}$ at $1 \mathrm{kHz}, 10 \mathrm{kHz}$ and $100 \mathrm{kHz}$, respectively. The thick solid black line in the figure is the magnetic field intensity of a whistler-mode wave actually observed in the shuttle experiment [4], which would be detectable by our loop antenna system above $500 \mathrm{~Hz}$. The rectangular regions represent typical magnetic field intensities of natural plasma waves in the magnetosphere: electromagnetic ion cyclotron waves (EMIC), magnetic noise bursts (MNB) and chorus emissions (for details, see for example [5]). The light gray curve represents the sensitivity of a typical search coil magnetometer on board satellites (in this case the one installed on the Mercury Magnetospheric Orbiter (MMO) to be launched in 2014 [6]). Though much less sensitive than the MMO search coils, our loop antenna system could observe these plasma waves at least when they become intense.

\section{Effect of MSEE Body on Sensor Performance}

Next, the effect of the MSEE body on the sensor performance was evaluated. As mentioned in Sect. 2, the MSEE
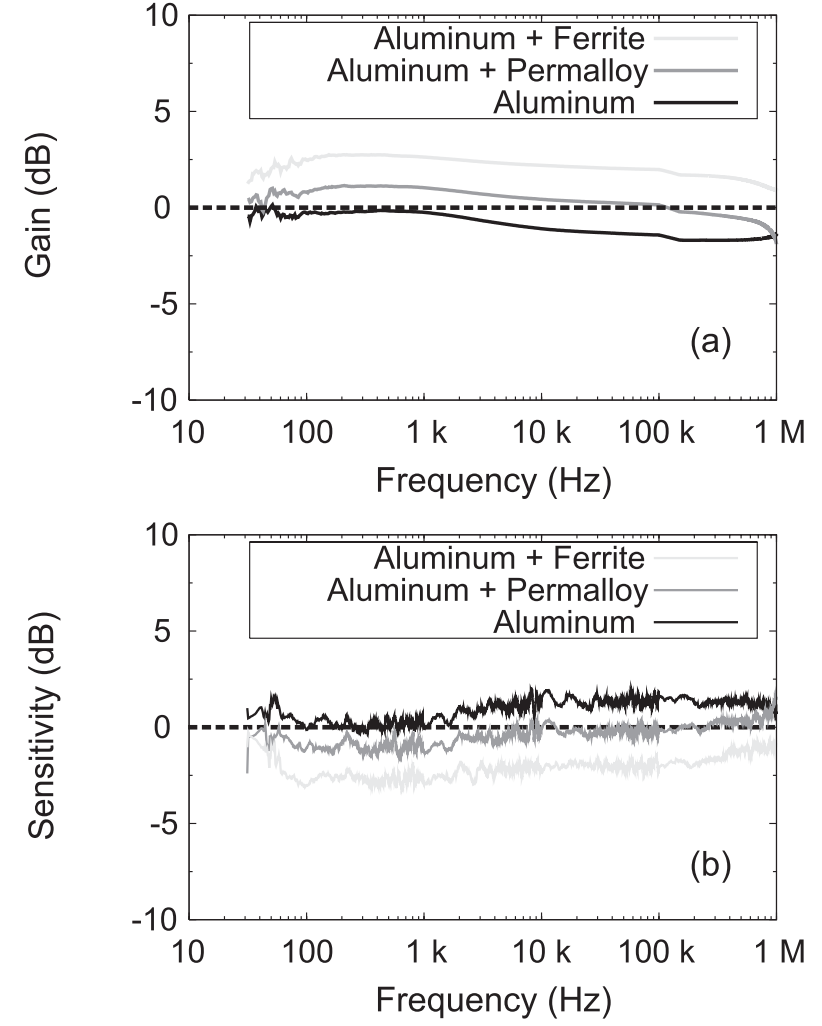

Fig. 4 Effects of MSEE body. (a) gain, and (b) sensitivity.

body consists of an aluminum chassis covered by magneticmaterial panels, which act as electrostatic and magnetic shielding, respectively. As shown in Fig. 2(a), the chassis consists of six panels of 1-mm-thick aluminum, which form the six surfaces of the cubic body. In order to avoid the creation of a shorted turn inside each loop antenna, slits are introduced between panels on adjacent surfaces, which are supported by a nonconductive (plastic) cubic frame. All six panels are nevertheless electrically connected to the common ground of the electronics, so as to act as an electrostatic shield. To reduce magnetic noise, a $0.2-\mathrm{mm}$ thick 45permalloy panel with high permeability $\left(\mu_{\mathrm{i}} \sim 2,400\right)$ or a 0.5 -mm thick ferrite composite panel $\left(\mu_{\mathrm{i}} \sim 100\right)$ is placed over each of the aluminum panels. These magnetic panels are also expected to reduce eddy-current loss on the surface of the aluminum panels when measuring an external magnetic field.

Figure 4 presents the deviations in gain and sensitivity for different shielding configurations, which are plotted relative to the values for the air-core loop without the MSEE body (i.e., the measured values in Fig. 3 are set as 0 -dB references). In Fig. 4(a), the black curve corresponds to the case where the MSEE body is just an aluminum chassis. The gain is seen to decrease appreciably at frequencies above $1 \mathrm{kHz}$, as the external magnetic flux is reduced by eddy currents induced on the aluminum surface. The case for the aluminum chassis covered by the permalloy panels is shown by the gray curve. The addition of the permalloy panels slightly mitigates the decrease in gain, as the high 
permeability of the permalloy reduces the penetration of the external magnetic field into the aluminum chassis, thereby reducing eddy-current induction. Note that the permalloy itself has a moderate conductivity leading to a small additional amount of eddy-current induction. At frequencies below $10 \mathrm{kHz}$, the gain is slightly enhanced compared to that for the air-core loop, since the permalloy panels act as a magnetic core that collects the magnetic flux crossing the loop area. As shown by the light gray curve, when the permalloy panels were replaced with ferrite composite panels, the gain was further enhanced by about $2 \mathrm{~dB}$ over the whole frequency range. This is due not only to the additional magnetic flux collected by the thicker panels, but also to a smaller amount of eddy-current induction in the panels because of their lower conductivity. The small ripples observed below $100 \mathrm{~Hz}$ on each curve are due to a decrease in the gain to almost the noise level at such low frequencies, as seen in Fig. 3(a).

Figure 4(b) plots the magnetic sensitivity, where the different curves correspond to those shown in Fig. 4(a). The rapid fluctuations are due to the thermal noise present during the measurements (see Fig. 3(b)). Compared to the case for the air-core $(0 \mathrm{~dB})$, the insertion of the aluminum chassis (black) causes a slight decrease in the sensitivity above $1 \mathrm{kHz}$, since additional thermal noise is generated due to the equivalent resistance associated with eddy-current loss on the aluminum surface. This is slightly mitigated (by $1.5 \mathrm{~dB}$ ) by the addition of the permalloy panels (gray), which reduces the eddy-current loss. Use of the ferrite composite panels (light gray) improves the sensitivity (by another $2 \mathrm{~dB}$ ) over the entire frequency range, since eddy-current loss is further mitigated.

On the other hand, even with the MSEE body inserted, the directionality of the loop antennas was found to exhibit a "figure-of-eight" pattern just as expected from theory. Over the frequency range of interest, the crosstalk between the three axes was also shown to be lower than $-40 \mathrm{~dB}$, which would lead to an error of just a few degrees when estimating the wave normal directions of plasma waves in geospace [7]. Thus, insertion of the MSEE body has an almost negligible effect on the performance of the antenna.

\section{Conclusion}

A compact loop antenna system to be used in an MSEE sensor node has been designed and fabricated, and its performance has been shown to be sufficient to allow measurement of the magnetic field activity around space structures in addition to intense natural plasma waves in geospace. In fact, the loop antenna system developed here is scheduled to be launched on board the JAXA (Japan Aerospace Exploration Agency) sounding rocket S-310-40 in 2011 to measure ionospheric propagation of LF and MF waves transmitted from ground stations. Though fine tuning of the antenna characteristics may be necessary for different electromagnetic phenomena, the loop antenna system is expected to contribute to the realization of an MSEE sensor network to be deployed for the study of space plasmas in the near future. Additionally, even on the ground, such a sensor network could be applied to flexible monitoring of the electromagnetic environment around electrical and electronic equipment. By measuring the electromagnetic field distribution generated around such equipment under actual operating conditions, the field sources can be identified using localization techniques [8]. Such a technique would be expected to be useful in a wide range of electromagnetic compatibility scenarios.

\section{Acknowledgment}

The authors would like to thank Mr. Y. Sato of JAXA and Mr. H. Takano of Meiwa System Company for their efforts in manufacturing the MSEE sensor node. We also thank Mr. Y. Sakamoto (now at Yokogawa Electric Corporation) and Mr. H. Tsukagoshi (now at Mitsubishi Electric Corporation) of Kanazawa University for their help with the design and measurement of the compact magnetic sensors. This study was supported in part by a Grant-in-Aid for Scientific Research (A) 19204048.

\section{References}

[1] D.A. Gurnett, "Principles of space plasma wave instrument design,' Measurement Techniques in Space Plasmas: Fields, Geophysical Monograph 103, R.F. Pfaff, J.E. Borovsky, and D.T. Young, eds., pp.121-136, American Geophysical Union, 1998

[2] H. Kojima, H. Fukuhara, Y. Mizuochi, S. Yagitani, H. Ikeda, Y. Miyake, H. Usui, H. Iwai, Y. Takizawa, Y. Ueda, and H. Yamakawa "Miniaturization of plasma wave receivers onboard scientific satellites and its application to the sensor network system for monitoring the electromagnetic environments in space," Advances in Geosciences, vol.21, pp.461-481, 2010.

[3] I.H. Cairns and D.A. Gurnett, "Plasma waves observed in the near vicinity of the space shuttle," J. Geophysical Research, vol.96, no.A8, pp.13,913-13,929, 1991.

[4] D.A. Gurnett, W.S. Kurth, J.T. Steinberg, P.M. Banks, R.I. Bush, and W.J. Raitt, "Whistler-mode radiation from the spacelab 2 electron beam," Geophys. Research Lett., vol.13, no.3, pp.225-228, 1986.

[5] H. Matsumoto, H. Kojima, Y. Omura, and I. Nagano, "Plasma waves in geospace: GEOTAIL observations," New Perspectives on the Earth's Magnetotail, Geophysical Monograph 105, pp.259-319, 1998.

[6] Y. Kasaba, J.-L. Bougeret, L.G. Blomberg, H. Kojima, S. Yagitani, M. Moncuquet, J.-G. Trotignon, G. Chanteur, A. Kumamoto, Y. Kasahara, J. Lichtenberger, Y. Omura, K. Ishisaka, and H. Matsumoto, "The Plasma Wave Investigation (PWI) onboard the BepiColombo/MMO: First measurement of electric fields, electromagnetic waves, and radio waves around Mercury," Planetary and Space Sciences, vol.58, no.1-2, pp.238-278, 2010.

[7] I. Nagano, M. Tsutsui, T. Okada, H. Kojima, H. Matsumoto, S Yagitani, and Y. Sato, "Characteristics of tri-axial search coil sensor on board the GEOTAIL spacecraft," IEICE Technical Report, AP94$100,1995$.

[8] S. Yagitani, K. Ishibana, I. Nagano, Y. Nishi, Y. Yoshimura, H Hayakawa, and K. Tsuruda, "Localization of low-frequency electromagnetic sources," Electronics and Communications in Japan, Part I: Communications, vol.88, no.6, pp.1-10, 2005. 